\title{
Gradientes de orientación en cercanías del borde de grano estudiados por EBSD
}

\author{
Orientation gradients in grain boundary \\ proximity studied by EBSD
}

\author{
Analía Roatta ${ }^{1,2}$, Claudio Schwindt ${ }^{3}$ \\ Natalia Soledad De Vincentis ${ }^{2}$, Javier Signorelli ${ }^{1,2}$
}

\author{
${ }^{1}$ Escuela de Formación Básica, Facultad de Ciencias Exactas, Ingeniería y Agrimensura, Universidad Nacional de Rosa- \\ rio - Av. Pellegrini 250, Rosario, Santa Fe, Argentina \\ e-mail: roatta@ifir-conicet.gov.ar; signorelli@ifir-conicet.gov.ar \\ ${ }^{2}$ División Materiales, IFIR-UNR-CONICET, Bv. 27 de Febrero 210b, Rosario, Santa Fe, Argentina. \\ e-mail: devincentis@ifir-conicet.gov.ar \\ ${ }^{3}$ Departamento de Ingeniería, Universidad Nacional del Sur - Av. Alem 1253, Bahía Blanca, Buenos Aires, Argentina. \\ e-mail: claudio.schwindt@uns.edu.ar
}

\section{RESUMEN}

El objetivo de este estudio es evaluar la desorientación local, su distribución y el gradiente de orientación en el desarrollo de la microestructura de una chapa de acero de calidad de embutido profundo (AKDQ). La microestructura se caracteriza con una resolución espacial a nivel de sub-grano usando difracción de electrones retrodifundidos (EBSD) en muestras sometidas a tensión uniaxial interrumpida en una probeta entallada y a solicitaciones típicas de procesos de conformado a través de ensayos Nakajima con diferentes geometrías de probeta. El empleo de la probeta entallada permite el estudio local de la evolución de la microestructura sobre un conjunto de granos identificables a medida que el material deforma, mientras las muestras correspondientes a los ensayos Nakajima se emplean para estudiar el comportamiento del gradiente de orientación con la distancia a los bordes de grano, bajo un enfoque estadístico. A partir de este último análisis se introducen dos nuevos parámetros basados en EBSD, llamados severidad promedio del gradiente (GAS) y espesor efectivo del borde (BET). En láminas de acero AKDQ de $0.67 \mathrm{~mm}$ de espesor, el valor del BET como indicador de la zona de influencia del BG resulta, en un sentido promedio, independiente del tamaño del grano y la condición de carga, sin superar los $3 \mu \mathrm{m}$. La severidad del gradiente muestra una buena correlación con la deformación plástica mesoscópica. Se observa además que la desorientación local se acumula en las proximidades de los bordes de grano mostrando diferencias en cada condición de carga.

Palabras clave: Gradiente de orientación, EBSD, Deformación plástica, Chapa de acero AKDQ, condiciones de deformación en plano.

\footnotetext{
ABSTRACT

The goal of this study is the evaluation of local misorientation, its distribution and orientation gradient in the microstructural development of a deep drawing quality steel sheet (AKDQ). The microstructure is characterized at sub-grain level spatial resolution using Electron Backscatter Diffraction (EBSD) in samples submitted to interrupted uniaxial tension in a notched sample and to typical solicitations in forming process through Nakajima tests with different sample geometries. The use of a notched sample allows performing a local study of the microstructural evolution in a set of identifiable grains as the material deforms, while the samples corresponding to Nakajima tests are used to study the behavior of the orientation gradient with the distance to the grain boundary under a statistical approach. From the latter analysis, two new EBSD-based parameters are introduced, named Gradient Average Severity (GAS) and Boundary Effective Thickness (BET). In $0.67 \mathrm{~mm}$ thick AKDQ steel sheets, the BET value as an indicator of GB influence zone is, in an average sense, independent on the grain size and the loading condition, below $3 \mu \mathrm{m}$. The gradient severity shows a good correlation with the mesoscopic plastic deformation. It is also observed that local misorientation accumulates in the grain boundary proximity, showing differences for each loading condition.
} 
Keywords: Orientation gradient, EBSD, Plastic deformation, AKDQ steel sheet, in-plane loading conditions.

\section{INTRODUCCIÓN}

Los procesos de deformación plástica, tales como el estirado y el embutido profundo, son en sí mismos procesos heterogéneos. Durante dichos procesos, se acumulan dislocaciones y generan rotaciones en la red cristalina, estableciéndose así desorientaciones locales. Numerosos trabajos analizan la dependencia de dichas desorientaciones con el tamaño de grano [1, 2], la forma de los granos [3] o las orientaciones cristalinas [4, 5]. SOIFER et al. [6] estudiaron la heterogeneidad de la deformación plástica en Cu policristalino, con tamaño de grano entre 5 y $50 \mu \mathrm{m}$, encontrando que la dureza aumentaba cerca de los bordes de grano en un factor 1.5 respecto del interior del grano, para una zona de borde de grano de $2 \mu \mathrm{m}$. SOER et al. [7] analizaron bicristales de Mo y observaron un efecto similar en la dureza cercana al borde de grano en una zona de aproximadamente $1 \mu \mathrm{m}$. CALCAGNOTTO et al. [8] reportaron gradientes de orientación en zonas cercanas a interfaces ferrita-martensita en aceros bifásicos. La presencia de gradientes de orientación tiene un rol de importancia en procesos de recristalización, fragmentación de granos y en la concentración de tensión en zonas cercanas a bordes de grano, que puede dar inicio a fracturas [9-12]. ABUZAID et al. [13] relacionaron la magnitud de la deformación plástica a través de los bordes de grano con el vector de Burgers residual, mientras que BIELER et al. [14] estudiaron la factibilidad de la transmisión de deslizamiento a través de bordes de grano según la alineación geométrica entre sistemas de deslizamiento activados a ambos lados del borde.

Actualmente, la difracción de electrones retrodifundidos (EBSD) permite obtener mapas precisos de orientaciones cristalinas y determinar desorientaciones relativas, resultando una herramienta de utilidad para la identificación y el estudio de zonas que presenten gradientes de orientación. La severidad de dichos gradientes, y por ende la heterogeneidad en la deformación plástica dentro de los granos, pueden determinarse a partir de varios parámetros [1,15-18], entre los cuales uno de los más usados es la desorientación promedio en núcleos, KAM (Kernel Average Misorientation) [5, 19]. Este índice, que resulta dependiente del tamaño de paso con el que se efectúa la medición [20,21], puede usarse para estimar densidad de dislocaciones geométricamente necesarias, compatible con el campo de tensiones presentes en el material [22,23]. Otro índice relacionado con la dispersión de orientaciones en el interior del grano es el GOS (Grain Orientation Spread); dicho parámetro ha sido estudiado por ALLAIN-BONASSO et al. [1] analizando el comportamiento de GOS/D (donde D es el tamaño de grano). Los autores focalizan el análisis en la influencia del tamaño de grano. KAMAYA et al. [24,25] establecieron un parámetro similar al KAM para la determinación de desorientación local, y observaron localización de desorientación en zonas cercanas a los bordes de grano en acero inoxidable tipo 316 deformado en tracción hasta 30\%. Recientemente, VACHHANI et al. [26] estudiaron aluminio policristalino y reportaron variaciones en el endurecimiento en una zona de $10 \mu \mathrm{m}$ desde los bordes de grano, relacionadas con la acumulación de dislocaciones.

El objetivo de este estudio es evaluar la desorientación local, su distribución y el gradiente de orientación en el desarrollo de la microestructura de un acero de calidad de embutido profundo (AKDQ). Las microestructuras analizadas se obtienen de muestras sometidas a tensión uniaxial interrumpida en una probeta entallada y a solicitaciones típicas de procesos de conformado a través de ensayos Nakajima con diferentes geometrías de probeta. Se realiza un estudio local de la evolución de la microestructura sobre un conjunto de granos identificables a medida que el material deforma, mientras que las muestras correspondientes a los ensayos Nakajima se emplean para estudiar el comportamiento del gradiente de orientación con la distancia a los borde de grano, bajo un enfoque estadístico. A partir de este último análisis se introducen dos nuevos parámetros basados en EBSD, llamados severidad promedio del gradiente (GAS) y espesor efectivo del borde (BET). Se discuten los resultados obtenidos y se evalúa la relación de los parámetros mencionados con la transmisión de deslizamiento a través de los bordes de grano.

\section{MATERIALES Y MÉTODOS}

\subsection{Descripción experimental}

El material utilizado en este trabajo es una chapa de acero electrogalvanizado de calidad de embutido (AKDQ) cuya composición química se presenta en Tabla 1 . La chapa tiene un espesor de $0.67 \mathrm{~mm}$, un tamaño de grano promedio de 9-10 (ASTM E122) y una dureza Vickers de 106HV5.

Los estados de carga, tensión uniaxial (UAT), deformación plana (PS) y equibiaxial (EBA) se obtuvieron mediantes ensayos Nakajima utilizando geometrías de probeta tipo "reloj de arena" con diferentes 
relaciones en sus dimensiones (Fig. 1). Las probetas se obtuvieron mediante corte laser de la chapa original.

Tabla 1: Composición química de la chapa de acero AKDQ (excluye el recubrimiento superficial).

\begin{tabular}{|c|c|c|c|c|c|c|c|c|c|c|c|}
\hline Elemento & $\mathbf{C}$ & $\mathbf{P}$ & $\mathbf{S}$ & $\mathbf{M n}$ & $\mathbf{S i}$ & $\mathbf{C r}$ & $\mathbf{N i}$ & $\mathbf{C u}$ & $\mathbf{M o}$ & $\mathbf{A l}$ & $\mathbf{N}(\mathbf{p p m})$ \\
\hline$\%$ peso & 0,070 & 0,020 & 0,009 & 0,300 & 0,040 & 0,020 & 0,010 & 0,010 & 0,010 & 0,192 & 88 \\
\hline
\end{tabular}

Desviación estándar $=0,001$

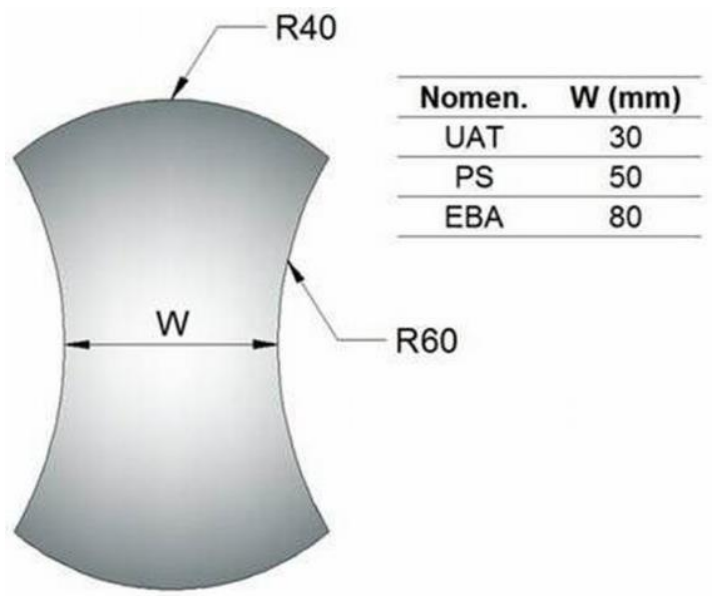

Figura 1: Detalle de la geometría de probeta utilizada en los ensayos Nakajima. La notación R40 y R60 hace referencia a radios de curvatura de $40 \mathrm{~mm}$ y $60 \mathrm{~mm}$, respectivamente.

De cada probeta se cortaron muestras de $3 \mathrm{~mm}$ x $5 \mathrm{~mm}$ en una región contigua a la zona con deformación localizada, pero sin abarcar el área con inestabilidad plástica. Para medir la deformación acumulada en el material se imprime, por deposición electroquímica sobre la superficie del material de partida, una grilla de círculos de $2.5 \mathrm{~mm}$ de diámetro. Después de ensayado el material, la medición de los ejes principales de las elipses resultantes permite determinar un valor aproximado de la deformación correspondiente a cada una de las muestras, obteniéndose deformaciones de $0.44,0.39$ y 0.80 para UAT, PS y EBA respectivamente. Asimismo, se tomaron muestras del material de partida (AR) como referencia.

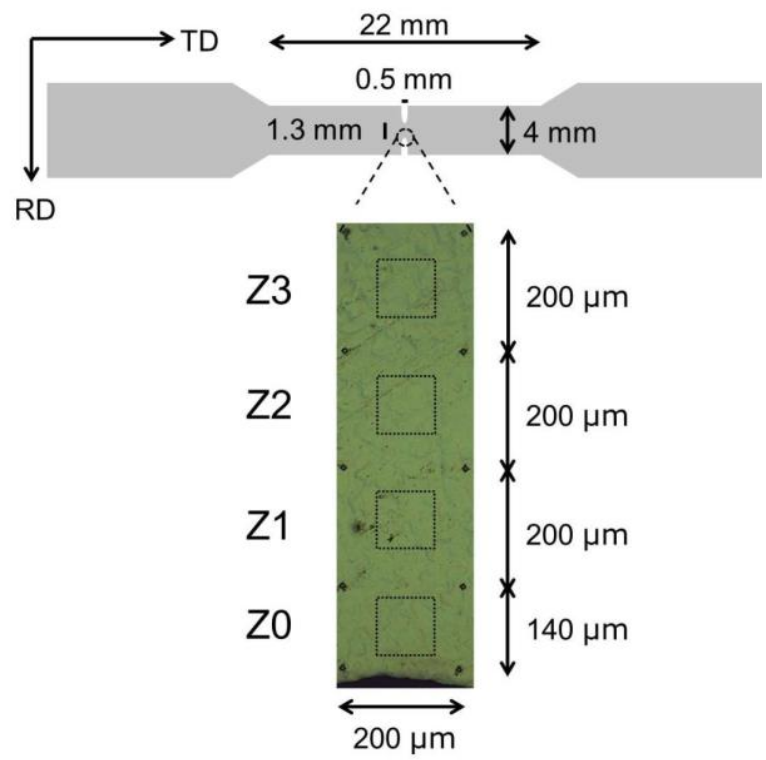

Figura 2: Esquema indicando las dimensiones de la probeta de tracción, las zonas estudiadas y posicionamiento de las indentas. 
Complementariamente, se cortó de la misma chapa una probeta de tracción, con una entalla profunda, tal como puede verse en la Fig. 2, con el eje longitudinal perpendicular a la dirección de laminación de la chapa con la finalidad de realizar un seguimiento de las desorientaciones cristalinas durante el ensayo. En este caso, debido a lo reducido de las dimensiones involucradas, la deformación mesoscópica se determinó mediante la medición de las posiciones relativas de un conjunto de indentas. Estas indentas permitieron también delimitar distintas zonas en la entalla para poder identificarlas a la hora de hacer un seguimiento de la microestructura en distintas etapas de deformación [27].

Los ensayos fueron desarrollados a temperatura ambiente utilizando una máquina de ensayo universal Instron 5989 de $150 \mathrm{kN}$ de capacidad, interrumpido a los $100 \mathrm{~s}$ y a los $300 \mathrm{~s}$ con una velocidad de desplazamiento del cabezal igual a $8.310^{-4} \mathrm{~mm} / \mathrm{s}$.

El conjunto de muestras fue analizado por medio de EBSD, empleando un microscopio electrónico de barrido FEI Quanta E con cañón emisor de campo. Se trabajó con una energía de $20 \mathrm{KeV}$ y a una distancia de trabajo de $15 \mathrm{~mm}$. La preparación de las muestras consistió en pulido en papel sólo para llevar la superficie a plano, luego pasta de diamante de 9, 6, 3 y $1 \mu \mathrm{m}$ y finalmente silica coloidal de $0.05 \mu \mathrm{m}$. Los barridos se efectuaron con tamaño de paso de $0.1 \mu \mathrm{m}$. En las muestras provenientes de los ensayos Nakajima se efectuaron 3 barridos por muestra de aproximadamente $65 \mu \mathrm{m}$ de lado cada uno, de modo de contar con una cantidad de granos suficiente como para un análisis estadístico, mientras que en la muestra correspondiente a la probeta con entalla se efectuó 1 barrido de aproximadamente $80 \mu \mathrm{m}$ de lado por zona y por cada etapa de interrupción del ensayo, efectuando el seguimiento de los mismos granos con el aumento de deformación.

\subsection{Modelo}

Numerosos trabajos relacionados con el estudio de desorientaciones intragranulares identifican distintas zonas en el interior de los granos, delimitadas por el comportamiento del gradiente de orientaciones. ALLAINBONASSO et al. [1] reportan que la desorientación decrece desde el borde de grano hacia el interior, y puede volver a crecer hacia el otro borde o fijarse en una meseta de muy bajo gradiente de orientación. El valor del gradiente y la distancia sobre la cual éste se extiende pueden ser diferentes a ambos lados de cada borde, como se muestra en la Fig. 3. Allí, la desorientación asignada a cada pixel es calculada a partir de la siguiente ecuación [24]:

$$
M_{L}^{(k)}(i)=\frac{1}{\# p t s .(k)} \sum_{j=1}^{\# p t s .(k)} m\left(i, j^{(k)}\right)
$$

donde $m$ es la desorientación entre el pixel $i$ (centro del kernel) y el $j$ (pixel del kernel de orden de vecindad $k)$.
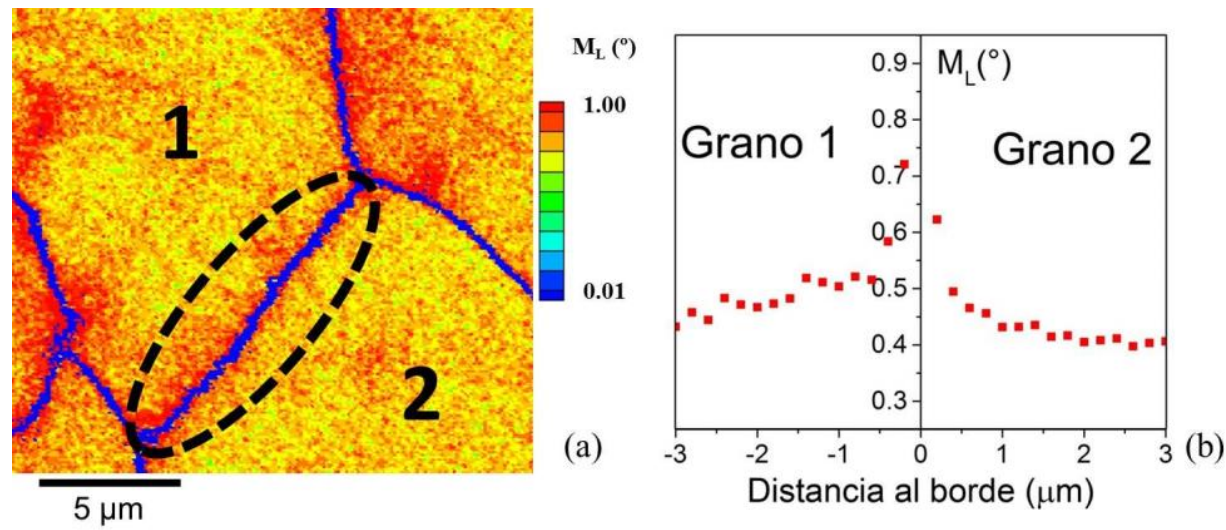

Figura 3: (a) Detalle del mapa de $\mathrm{M}_{\mathrm{L}}$ (escala logarítmica) del barrido correspondiente a $\mathrm{Z} 2$ luego del ensayo de $300 \mathrm{~s}$ y (b) gráfica del perfil promedio de $\mathrm{M}_{\mathrm{L}}$ a ambos lados del borde resaltado.

Cada punto en la gráfica de Fig. 3 (b) corresponde a un promedio de desorientaciones en bins de 0.2 $\mu \mathrm{m}$ de espesor a partir del borde de grano. De esta forma, en cada grano se identificarían dos zonas: el "borde", que en realidad hace referencia no sólo al borde de grano propiamente dicho sino a su zona de influencia 
hacia el interior del grano, y que se caracteriza por un gradiente negativo de desorientación, y el "núcleo" o zona central del grano, de gradiente aproximadamente nulo (Fig. 4).

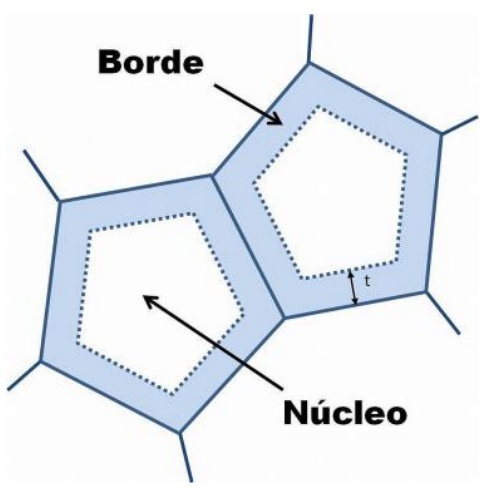

Figura 4: Esquema de las zonas de "borde" y "núcleo" de los granos.

Sólo dos parámetros son suficientes para esta caracterización: $\lambda$, la distancia de influencia del borde mencionada, y $\beta$, la severidad del gradiente, donde $\operatorname{la} \operatorname{tg}(\beta)$ es medida en $\% \mu \mathrm{m}$. Para evaluar ambos valores, se ajustaron los datos de desorientaciones con dos rectas, una de pendiente negativa y otra de pendiente nula, variando la cantidad de puntos en el ajuste de cada una hasta obtener el mínimo error, en el sentido de mínimos cuadrados. Debe tenerse en cuenta que, debido a que los datos de partida corresponden a análisis de EBSD en 2D, y por ende se desconoce la orientación del plano de borde de grano, los valores de $\beta$ y $\lambda$ obtenidos corresponden a una subestimación y sobreestimación de los valores reales, respectivamente.

\section{RESULTADOS}

En la siguiente sección se detallarán los resultados obtenidos para ambos grupos de muestras. Primeramente, se realizará un análisis local manteniendo trazabilidad de las orientaciones cristalinas; y a continuación se presentará un estudio de carácter estadístico sobre un número importante de bordes de grano provenientes de las probetas sometidas a los ensayos Nakajima.

\subsection{Análisis local}

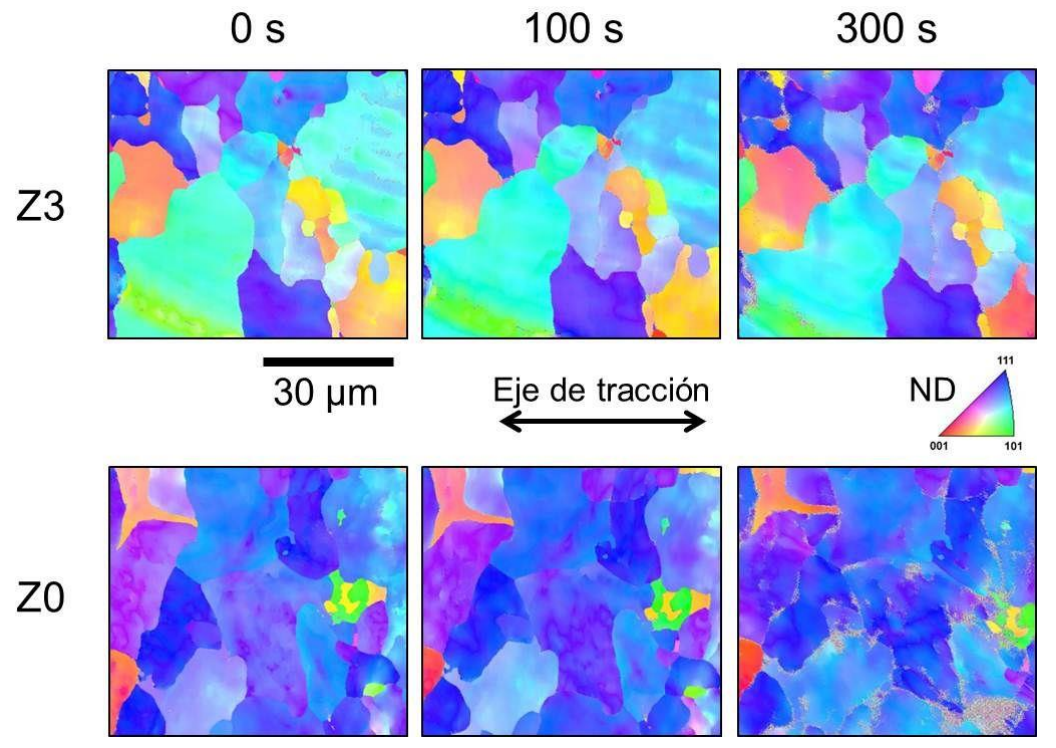

Figura 5: Mapas de figura de polos inversa de las zonas Z3 y Z0 de la probeta de tracción.

En esta sección se muestran los resultados obtenidos para la probeta de tracción entallada, particularmente en las zonas Z3 y Z0, más próximas al eje de la probeta y a la punta de la entalla, respectivamente. El 
diseño y dimensión de la probeta permitió establecer distintos parámetros de control del proceso de deformación a la que fue sometida:

- Control de la zona donde se desarrolla la mayor deformación debido a la presencia de la entalla.

- Variación del estado de carga en cada zona, aproximándose a tracción simple a medida que nos acercamos al eje de la probeta (Z3).

- Interrupción del ensayo y medición por EBSD del mismo conjunto de granos luego de cada etapa. La dimensión reducida de la probeta no requiere el corte de la misma.

La delimitación de cada zona analizada es de aproximadamente $80 \mu \mathrm{m}$ de lado en el estado inicial, y fue variada posteriormente de modo de efectuar un seguimiento de los mismos granos. En la Fig. 5 se observan los mapas de figura de polos inversa (IPF) obtenidos mediante EBSD para las zonas Z3 y Z0 al inicio y luego de 100 s y 300 s de duración del ensayo. La deformación efectiva de Von Mises correspondiente a cada zona, estimada a partir del desplazamiento relativo de las indentas, se muestra en la Fig. 6. Se observa una deformación aproximadamente uniforme luego de $100 \mathrm{~s}$, variando entre 4 y 7\%; mientras que a los $300 \mathrm{~s}$ la zona más cercana al borde de la entalla (Z0) muestra un 17\% de deformación comparada con solo un $7 \%$ en el centro (Z3).

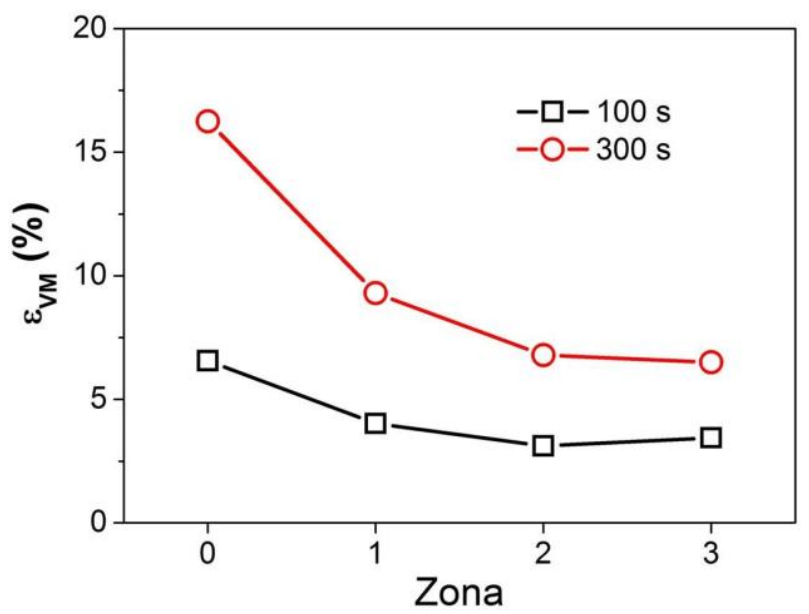

Figura 6: Deformación de Von Mises obtenida en cada zona.

A partir de cada mapa de orientaciones cristalinas se calculó la desorientación local (Ec. 1, Fig. 7). A simple vista se observa que la proporción de áreas de alta desorientación es similar para ambas zonas hasta 100 s. Las mayores desorientaciones ocurren principalmente en la zona cercana a los bordes de grano. A 300 s la desorientación se incrementa considerablemente en la zona Z0 con valores altos de desorientación tanto en los bordes como en el interior del grano. Se observa especialmente en la esquina inferior derecha de la zona Z0 a 300 s (Figs. 5 y 7) una degradación de las mediciones, compatible con el grado de deformación. Por este motivo, se tuvo especial cuidado en esta zona en la selección de los bordes a ser analizados. En la Fig. 7 se indican con letra imprenta mayúscula los granos elegidos para el análisis y sus primeros vecinos. 


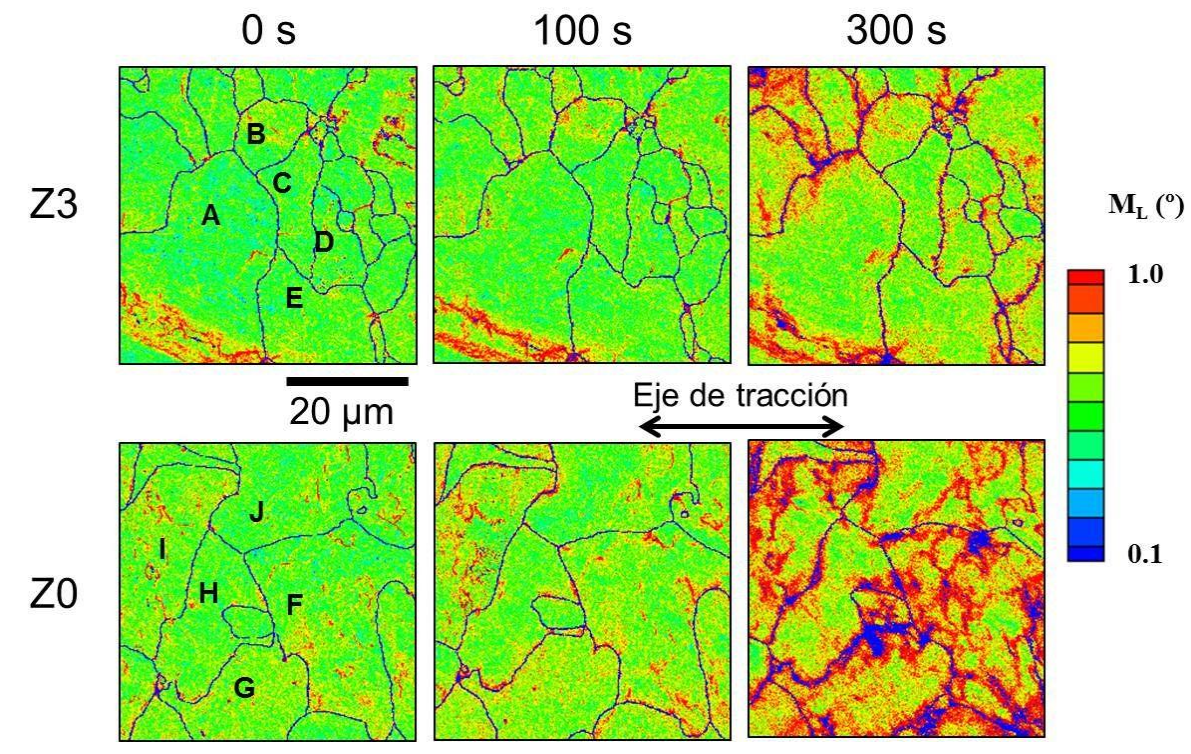

Figura 7: Mapas de $\mathrm{M}_{\mathrm{L}}$ (escala logarítmica) obtenidos en las zonas $\mathrm{Z3}$ y Z0 en las tres etapas, indicando los granos cuyos bordes se analizan. Los bordes de grano están marcados con líneas llenas.

La Fig. 8 muestra los perfiles de desorientación obtenidos para los bordes del grano C de la zona Z3 y la Tabla 2, los correspondientes valores de permeabilidad $\chi=\cos (\boldsymbol{\kappa}) \cos (\psi)$ ( $\psi$ es el ángulo entre las direcciones normales a los planos de deslizamiento y $\kappa$ el ángulo entre las direcciones de deslizamiento a ambos lados de un borde de grano) al inicio y en las dos etapas posteriores del ensayo.
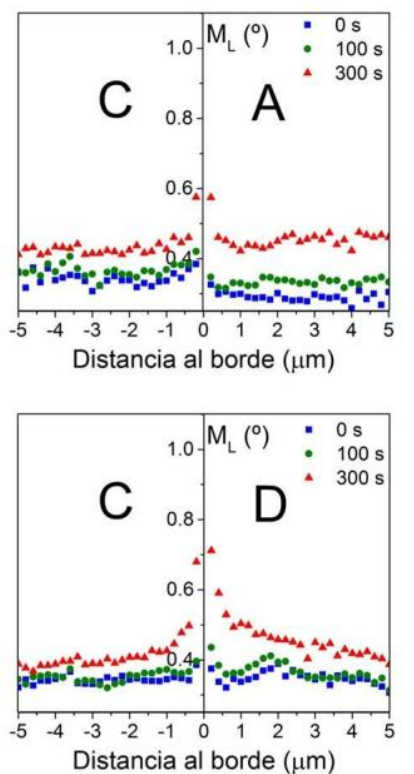

(a)

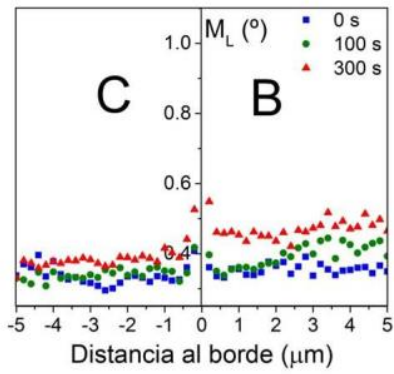

(b)

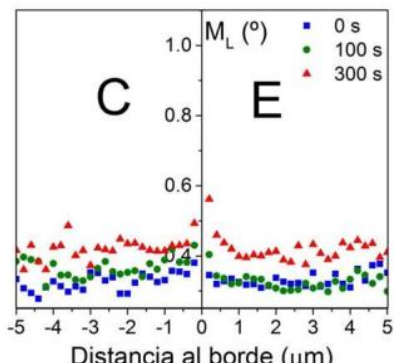

(d)

Figura 8: Perfiles de desorientación en los bordes seleccionados de la zona Z3.

Tabla 2: Valores de permeabilidad $\chi$ obtenidos para los bordes seleccionados de la zona Z3.

\begin{tabular}{|c|c|c|c|c|}
\hline ETAPA & C-A & C-B & C-D & C-E \\
\hline $0 \mathrm{~s}$ & 0.98 & 0.87 & 0.87 & 0.81 \\
\hline $100 \mathrm{~s}$ & 0.97 & 0.87 & 0.87 & 0.82 \\
\hline $300 \mathrm{~s}$ & 0.97 & 0.86 & 0.87 & 0.83 \\
\hline
\end{tabular}


Mientras mayor sea el valor de $\chi$, mayor será la "permeabilidad" del borde. La determinación de $\chi$ se realizó buscando en primer lugar los planos de deslizamiento que presentan menor ángulo $\psi$ y luego las direcciones que minimizan el ángulo $\kappa$. Los valores de $\mathrm{M}_{\mathrm{L}}$ obtenidos permiten inferir una incerteza en la permeabilidad menor a 0.04 considerando una dispersión máxima de $1^{\circ}$ en el valor de las orientaciones promedio sobre la región contigua al borde de grano. Frente al borde con el grano A se observan severidad y ancho de influencia similares a ambos lados del borde, acompañados con altos valores de permeabilidad en todas las etapas. En el borde C-B se observa un incremento uniforme en la desorientación del lado B cuando la deformación alcanza aproximadamente el 7\% superando la desorientación de C (Fig. 8 (b)). Excepto el punto correspondiente a la medición ocurrida inmediatamente a la derecha del borde, los demás valores pueden ajustarse con una recta horizontal, lo cual implicaría que forman parte del núcleo del grano, y entonces este borde se identificaría como de muy baja influencia. A diferencia de los bordes anteriores, en C-D se observa claramente la formación de gradientes de desorientación con severidades similares de ambos lados, pero con un ancho de influencia mayor del lado del grano D. En este caso el parámetro $\chi$ es relativamente bajo, lo cual sugiere una baja permeabilidad y puede estar relacionado con la alta severidad del borde (Fig. 8 (c)). Puede apreciarse también que la desorientación del grano $\mathrm{D}$ es levemente mayor que en el grano $\mathrm{C}$ en la última etapa del ensayo. El borde C-E, presenta un comportamiento menos uniforme con mayor severidad del lado del grano E (Fig. 8 (d)).

Los perfiles obtenidos en los bordes de grano seleccionados en la zona Z0 se presentan en la Fig. 9. La Tabla 3 contiene los correspondientes valores de permeabilidad $\chi$ al inicio y en las dos etapas posteriores del ensayo. El grado de desorientación a $300 \mathrm{~s}$ supera ampliamente a los obtenidos en la zona Z3, además de representar un salto importante con respecto a la propia zona Z0 en la etapa de $100 \mathrm{~s}$, lo que es compatible con un notable aumento de la deformación. Los bordes seleccionados presentan particularidades diferentes entre sí, que permiten mostrar la versatilidad del modelo aplicado.
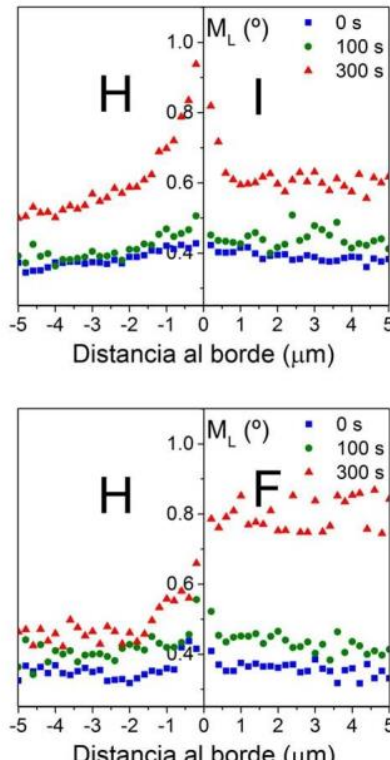

Distancia al borde $(\mu \mathrm{m})$ (a)

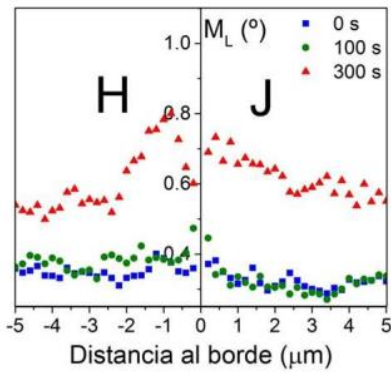

(b)

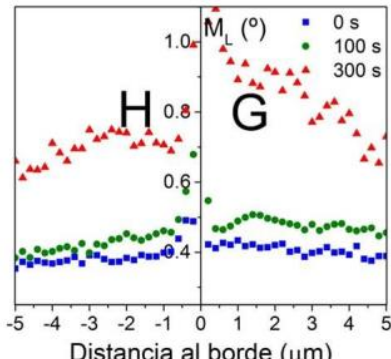

(d)

Figura 9: Perfiles de desorientación en los bordes seleccionados de la zona Z0.

Tabla 3: Valores de permeabilidad $\chi$ obtenidos para los bordes seleccionados de la zona Z0.

\begin{tabular}{|c|c|c|c|c|}
\hline ETAPA & H-I & H-J & H-F & H-G \\
\hline $0 \mathrm{~s}$ & 0.95 & 0.98 & 0.75 & 0.91 \\
\hline $100 \mathrm{~s}$ & 0.96 & 0.98 & 0.75 & 0.84 \\
\hline $300 \mathrm{~s}$ & 0.64 & 0.97 & 0.67 & 0.97 \\
\hline
\end{tabular}

El borde H-I muestra alta severidad a ambos lados (con mayor valor en I), y una zona de influencia mayor del lado H. Este resultado implica alta localización de desorientaciones en las zonas próximas al borde de grano de ambos lados. Además el factor $\chi$ decrece fuertemente en la última etapa de deformación, lo que 
refuerza la idea de acumulación de dislocaciones en dichas zonas a causa de la baja permeabilidad de ese borde. El borde H-J, en cambio, mantiene un alto factor $\chi$, lo que lleva a pensar que la mayor permeabilidad de este borde frente a la transmisión de deslizamiento dificulta la formación de fuertes gradientes de orientación. Se puede ver del lado H (Fig. 7) una zona de localización de orientaciones alejada del borde en algo más de $1 \mu \mathrm{m}$ que no puede describirse mediante el perfil simple empleado y que surge en la etapa final de deformación, por lo que es necesario analizar bajo qué condiciones puede considerarse válido el modelo estudiado. Del lado J, todos los puntos responden a una recta decreciente, correspondiendo a un ancho de influencia del borde en este grano mayor a $5 \mu \mathrm{m}$. El borde H-F muestra un comportamiento bastante particular al aumentar la deformación; hasta la etapa de $100 \mathrm{~s}$, ambos lados del borde presentan similar severidad y distancia de influencia, pero luego de $300 \mathrm{~s}$ aparece una marcada severidad del lado $\mathrm{H}$, y una alta desorientación del lado F. Parece ser que en este grano las desorientaciones no se acumulan solamente en el borde de grano sino que también en su interior, por lo que la distinción entre las dos partes del grano (borde y núcleo) desaparece. Respecto al factor $\chi$, mantiene un valor bajo de 0.75 hasta la etapa de 100 s y decrece en la etapa final. Finalmente, se analiza el borde $\mathrm{H}-\mathrm{G}$, de alta permeabilidad, que presenta comparativamente las mayores desorientaciones; alta severidad y bajo espesor del lado $\mathrm{H}$ y desorientaciones suavemente decrecientes del lado $\mathrm{G}$, indicando una distancia de influencia efectiva mayor a los $5 \mu \mathrm{m}$. El rasgo común de todos los perfiles analizados es que las desorientaciones en el núcleo del grano no superan los $0.5^{\circ}$ hasta niveles de deformación de $\sim 7 \%$.

\subsection{Análisis global}

En esta sección se analizan las muestras resultantes de los ensayos de Nakajima. En cada una de las muestras se realizaron varios barridos de EBSD de distintos tamaños, permitiendo así el análisis de 3069 bordes de grano. La Fig. 10 muestra mapas típicos de figura de polos inversa de dichas muestras, efectuados con un paso de $0.1 \mu \mathrm{m}$, donde puede observarse el desarrollo de mayor heterogeneidad de las orientaciones con el grado de deformación que alcanza el material.

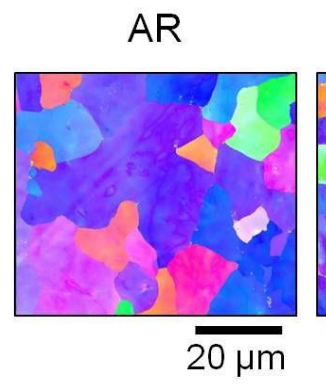

UAT

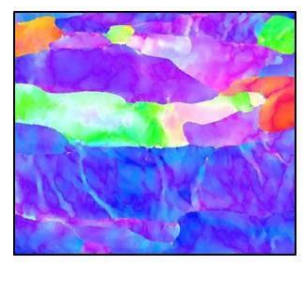

PS

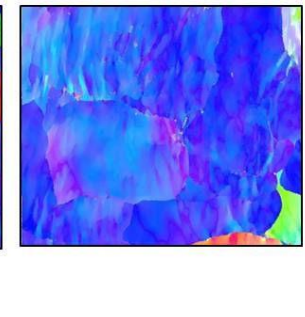

EBA

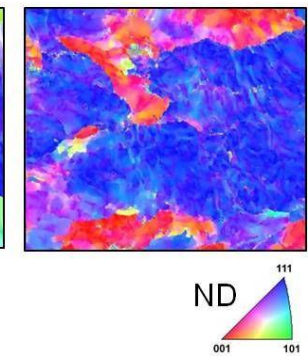

Figura 10: Mapas de figura de polos inversa obtenidos para el material recibido (AR) y luego de los diferentes caminos de deformación. ND se encuentra orientada perpendicular al papel.

Analizando globalmente los diversos mapas de orientaciones medidos se obtiene que el promedio de la desorientación local en todos los bordes de grano independientemente del modelo descripto, y normalizada respecto al valor en el material de partida, efectivamente decrece desde el borde de grano hacia el interior, y se estabiliza en valores de desorientaciones finales que sí parecen estar relacionadas con el tipo y la intensidad de deformación alcanzados (Fig. 11). El comportamiento de la desorientación promedio en la zona próxima al borde de grano difiere para el ensayo EBA respecto a PS y UAT. Mientras que en estos últimos la desorientación siempre aumenta al acercarse al borde, en el caso equibiaxial la zona de mayor heterogeneidad se separa del borde en $\sim 0.5 \mu \mathrm{m}$, resultado que se manifiesta claramente aún dentro de la dispersión estadística de las mediciones (indicada con barras en la Fig. 11). Debe notarse que en los análisis por EBSD se descartaron los granos de tamaño menor a 100 pixels.

Un análisis local, borde a borde, reveló una variación importante entre los perfiles de desorientaciones por lo que se decidió entonces determinar qué proporción de los bordes de granos analizados pueden ser caracterizados correctamente por el perfil bilineal simple descripto en la sección anterior. El criterio definido para considerar aceptable el ajuste fue que los coeficientes de correlación $R_{1}$ y $R_{2}$ para cada segmento del perfil cumplan las condiciones $\mathrm{R}_{1}<-0.5$ y $\left|\mathrm{R}_{2}\right|>0.5$ respectivamente. Los resultados obtenidos aplicando dicho criterio arrojaron que el modelo resultó válido para el $25 \%$ de los bordes de grano estudiados, siendo además similar la proporción para los tres caminos de deformación empleados. Este valor demuestra el carácter alta- 
mente local y complejo del comportamiento de la desorientación entre el centro del grano, los bordes y granos vecinos.
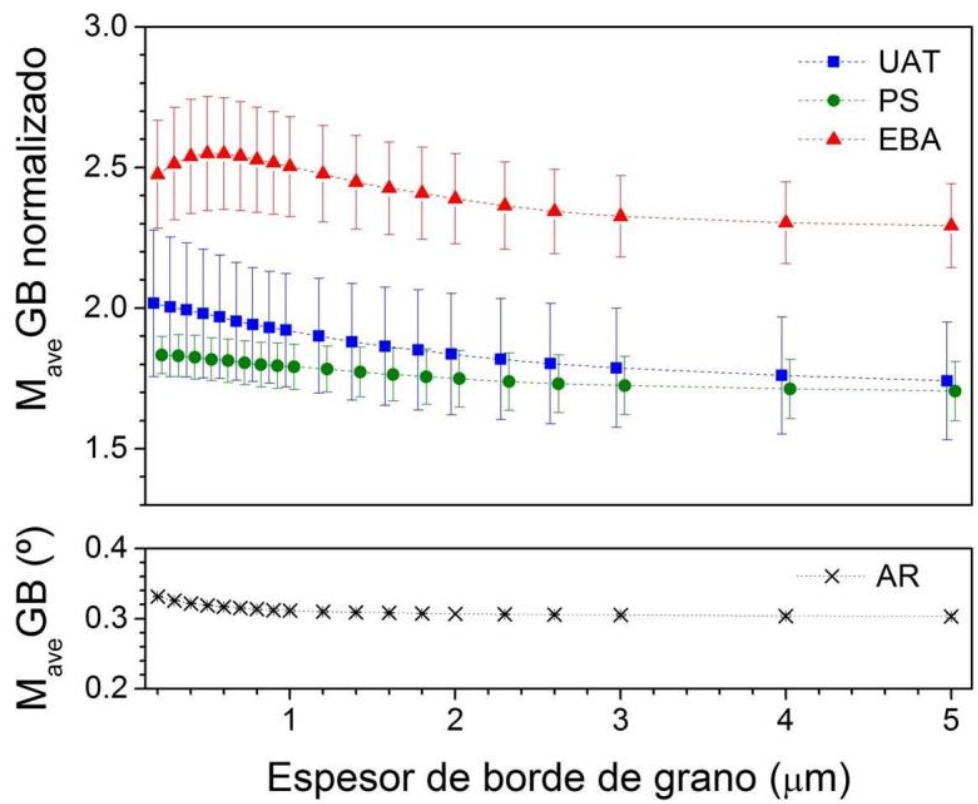

Figura 11: Gráfica del promedio de desorientaciones locales sobre regiones adyacentes a los bordes de grano en función del ancho de dichas regiones.

Tomando en cuenta sólo los bordes de grano que cumplen con las características que responden a los criterios mencionados, se grafican en la Fig. 12 los histogramas de $\lambda$ y $\lambda / \mathrm{D}$ (D es el diámetro de grano) para dos grupos de granos divididos según pertenezcan al 50\% de mayor o de menor valor de $\mathrm{D}$. Del histograma de la derecha se observa una tendencia a que $\lambda$ presente sus valores más probables en torno al $10 \%$ del diámetro de grano, como fue mencionado por ALLAIN-BONASSO et al. [1].

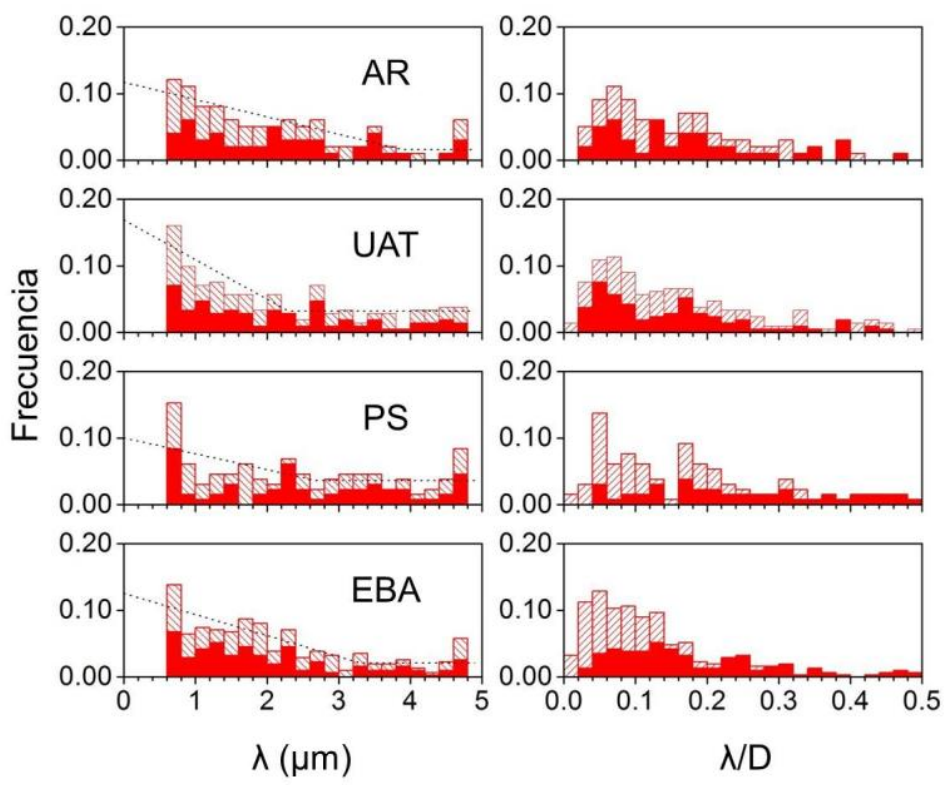

Figura 12: Distribución del ancho efectivo de bordes de grano $\lambda$ (a) y del valor escalado $\lambda / D$ (b) para los bordes de grano analizados. Las barras rellenas corresponden al 50\% de los granos más pequeños, mientras que el 50\% restante se representa con barras rayadas. 
Respecto del histograma de la izquierda de la figura, la forma de la distribución de $\lambda$ es similar para los distintos caminos de deformación, siendo en general decreciente desde el borde del grano hacia el centro. Puede apreciarse que la probabilidad resulta mucho menos sensible a la distancia al borde de grano una vez que alcanza un valor de distancia particular, que se definirá como "espesor efectivo de borde" o BET (Boundary Effective Thickness). La aplicación de un ajuste bilineal produce valores de BET iguales a $3.9 \mu \mathrm{m}$ para la muestra AR, $2.3 \mu \mathrm{m}$ para UAT, $2.7 \mu \mathrm{m}$ para PS y $3.3 \mu \mathrm{m}$ para EBA. Si bien las distribuciones de $\lambda$ para ambas poblaciones en cada muestra son similares, se observa que en los histogramas de $\lambda / \mathrm{D}$ las distribuciones de granos más pequeños está levemente corrida hacia valores mayores que la distribución complementaria; esto podría estar ocurriendo debido a que en los granos más grandes la zona interior del grano resultaría influenciada en menor medida por las características del borde. Estos resultados concuerdan con los resultados reportados por SCHERIAU et al. [2] sobre subdivisión de granos como resultado de deslizamiento cerca de bordes de grano.

A continuación se analiza la severidad en la desorientación de dichos bordes, descripta por el parámetro $\beta$. Los valores obtenidos se presentan en la Fig. 13 (a), donde se observa que sus distribuciones se desplazan hacia valores mayores a medida que aumenta la deformación plástica desarrollada en la muestra. Los valores de $\beta$ correspondientes al máximo de cada distribución se grafican en función de la deformación (Fig. 13 (b)), mostrando una relación lineal que permitiría el uso de este parámetro como una medida de la deformación mesoscópica de la muestra. Este ángulo será llamado "severidad promedio de gradiente" o GAS (Gradient Average Severity), y debe ser tenido en cuenta siempre en conjunto con el parámetro BET ya que ambos son necesarios para caracterizar en promedio la zona de influencia de los bordes de grano en el material.
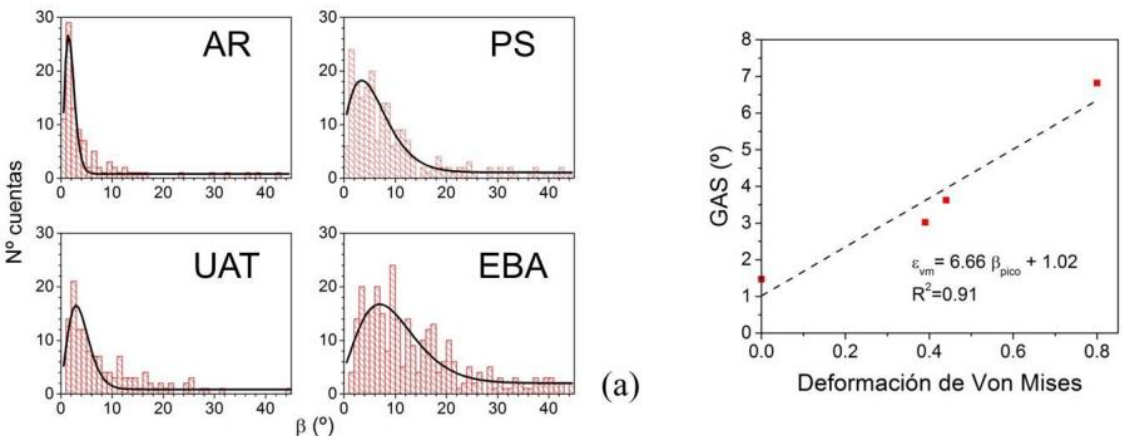

Figura 13: (a) Distribución de los valores de $\beta$ obtenidos para todas las muestras y (b) correlación entre los valores donde ocurre el máximo de dichas distribuciones y la deformación plástica medida.

\section{DISCUSIÓN}

El conjunto de ensayos llevados a cabo para el presente trabajo permitió caracterizar, tanto de forma local como global, los gradientes de desorientación desarrollados en las zonas cercanas a los bordes de grano para distintos tipos y grados de deformación plástica. Pueden efectuarse varias observaciones sobre los resultados obtenidos, tanto sobre el desarrollo de gradientes de orientación en zonas de bordes de grano como sobre el perfil para caracterizarlos. Dichas observaciones se presentan a continuación, junto con un análisis del respaldo bibliográfico y la propuesta de trabajo a futuro.

En primer lugar, es necesario discutir la viabilidad del modelo utilizado para describir las desorientaciones en zonas de bordes de grano. Varios autores han observado variación en desorientación y en deformación en dichas zonas; entre ellos, ALLAIN-BONASSO et al. [1] propusieron la representación utilizada en el presente trabajo. A partir de los bordes particulares analizados en la Sección 3.1 puede observarse que dicha representación es capaz de describir la mayoría de las situaciones analizadas, tanto cuando la desorientación decrece rápida o lentamente hacia el interior del grano, o cuando prácticamente no varía, casos en los que podría hablarse de escasa influencia de dicho borde en el interior del grano. El borde en particular que no cumple con este perfil simple (H-J a 300 s en la Fig. 9 (b)), muestra que la desorientación presenta valores máximos en una región distante del borde de grano de aprox. $1 \mu \mathrm{m}$ y decrece tanto hacia el borde de grano como hacia el interior. Al analizar el mapa de orientaciones (Fig. 5) se observa que efectivamente hay una localización de la orientación del lado $\mathrm{H}$ de dicho borde y que además se trata de un borde de grano de menos de $10 \mu \mathrm{m}$ de longitud, en el cual la cercanía de los puntos triples podría influir en el desarrollo de las desorientaciones. Es de notar que esta particularidad recién se manifiesta en la etapa final del ensayo. Otro 
factor a considerar, teniendo en cuenta estudios previos de SUBEDI et al. [19], es el tamaño de paso de medición por EBSD.

En segundo lugar, pero a modo de complemento de lo expuesto en el párrafo anterior, debe pensarse en la aplicabilidad del modelo para el análisis de datos en gran escala. En la Sección 3.2 se analizaron los bordes de grano en muestras para ensayos Nakajima. Los distintos caminos de deformación impuestos no parecen haber afectado en gran medida el espesor de influencia de los bordes de grano, ya que el valor más probable para cada muestra ronda los $3 \mu \mathrm{m}$ y además resulta independiente del tamaño del grano que participa. Diferente fue la situación de la severidad, que aumenta con el nivel de deformación. Este resultado indicaría que una mayor deformación introduce desorientaciones más severas en las zonas de bordes de grano pero manteniéndose el espesor de influencia. La introducción de los parámetros BET y GAS contribuye a la caracterización en promedio de los gradientes de bordes de grano para cada muestra analizada.

Teniendo en cuenta los resultados obtenidos en los dos grupos de muestras estudiados, mientras que las correspondientes a ensayos Nakajima presentan alto nivel de deformación plástica (hasta 0.8), en la probeta de tracción entallada, en cambio, se desarrollan deformaciones mucho menores, incluso en la zona cercana al borde de la entalla donde se alcanza aproximadamente 0.17. Puede decirse que el nivel de deformación impuesta al material parece influir en la aplicabilidad del modelo, ya que éste falla para describir algunos bordes en la zona más exigida de la probeta entallada (zona Z0 en la última etapa de deformación) y sólo explica $25 \%$ de los casos en las probetas Nakajima. El hecho de obtenerse también este porcentaje en la muestra sin deformar puede deberse al bajo gradiente presente en las cercanía de sus bordes, que dificulta el ajuste efectuado.

En el análisis de bordes particulares, se observó que la desorientación desarrollada en un mismo grano pero en cercanías de cada uno de sus bordes de grano es diferente para cada vecino, lo cual refuerza el carácter local de la estructura de desorientaciones desarrollada.

Finalmente, se discute la posibilidad de la existencia de una relación entre la severidad y espesor de bordes y el factor de permeabilidad $\chi$. De acuerdo a los resultados obtenidos en los bordes analizados, no se observa una correlación directa entre ambos parámetros, ya que en algunos casos el factor $\chi$ es alto tanto cuando se observa similar espesor y severidad a ambos lados de un borde, como en bordes con características diferentes a ambos lados. Esta situación puede deberse a varios factores: se desconoce en qué planos de deslizamiento específicos se desarrolla la deformación en cada grano, siendo el factor $\chi$ solo un indicador de los sistemas de deslizamiento con potencialmente mejor permeabilidad, y además la medición $2 \mathrm{D}$ de los mapas de orientaciones permite evaluar únicamente una proyección del plano de borde de grano. Esta información resulta de gran importancia para la determinación de factores de permeabilidad más realistas [14] y requiere para su evaluación la implementación de otras técnicas experimentales como FIB (Focused Ion Beam) o recurrir a métodos de modelización.

\section{CONCLUSIONES}

El análisis de orientaciones a través de EBSD permitió caracterizar la heterogeneidad en la localización de la desorientación originada durante la deformación plástica en muestras de un acero sometido a diferentes caminos de deformación: tensión uniaxial, equibiaxial y deformación plana.

A nivel local, mediante el seguimiento del campo de orientaciones se caracterizó la zona de influencia de bordes particulares en distintas regiones de una probeta con entalla profunda. Se encontró que la severidad aumenta en los bordes de las zonas más deformadas (Z0), y el comportamiento de la desorientación local en ambos lados de los bordes no muestra una correlación directa con el factor de permeabilidad en borde de grano.

A nivel mesoscópico, se realizó el estudio de un número importante de bordes de grano mediante las muestras extraídas de las probetas resultantes de los ensayos Nakajima. Se analizó el comportamiento del gradiente de orientación con la distancia a los borde de grano, bajo un enfoque estadístico. El promedio de la desorientación local en todos los bordes de grano decrece desde el borde de grano hacia el interior, y se estabiliza en valores de desorientaciones finales relacionadas con el tipo y el grado de deformación alcanzados. En la zona próxima al borde de grano, el ensayo EBA presenta su mayor heterogeneidad separada del borde en $\sim 0.5 \mu \mathrm{m}$. Una evaluación de las causas de este efecto podrían responderse con mediciones a partir de ensayos EBA a menor deformación. Asimismo, se introdujeron dos nuevos parámetros: el ancho efectivo del borde y la severidad promedio del gradiente. El BET determinado en estas muestras fue cercano a $3 \mu \mathrm{m}$, tanto para la muestra original como para los distintos caminos de deformación. El GAS presentó buena correlación con la deformación plástica mesoscópica. 


\section{AGRADECIMIENTOS}

Los autores agradecen el financiamiento provisto por ANPCyT (Agencia Nacional de Promoción Científica y Tecnológica) - PICT 2013-0558.

\section{BIBLIOGRAFÍA}

[1] ALLAIN-BONASSO, N., WAGNER, F., BERBENNI, S., et al., "A study of the heterogeneity of plastic deformation in IF steel by EBSD", Materials Science and Engineering A, v. 548, pp. 56-63, Jun. 2012.

[2] SCHERIAU, S., PIPPAN, R., "Influence of grain size on orientation changes during plastic deformation", Materials Science and Engineering A, v. 493, pp. 48-52, Oct. 2008.

[3] FRANCIOSI, P., BERBENNI, S., "Multi-laminate plastic-strain organization for non-uniform TFA modeling of poly-crystal regularized plastic flow", International Journal of Plasticity, v. 24, pp. 1549-1580, Sep. 2008.

[4] KANJARLA, A.K., VAN HOUTTE, P., DELANNAY, L., "Assessment of plastic heterogeneity in grain interaction models using crystal plasticity finite element method", International Journal of Plasticity, v. 26, pp. 1220-1233, Aug. 2010.

[5] ROLLETT, A.D., WAGNER, F., ALLAIN-BONASSO, N., et al., "Comparison of Gradients in Orientation and Stress between Experiment and Simulation", Materials Science Forum, v. 702-703, pp. 463-468, Jan. 2012.

[6] SOIFER, YA. M., VERDYAN, A., KAZAKEVICH, M., et al., "Nanohardness of copper in the vicinity of grain boundaries", Scripta Materialia, v. 47, pp. 799-804, Dec. 2002.

[7] SOER, W.A., AIFANTIS, K.E., DE HOSSON, J.TH.M., "Incipient plasticity during nanoindentation at grain boundaries in body-centered cubic metals", Acta Materialia, v. 53, pp. 4665-4676, Oct. 2005.

[8] CALCAGNOTTO, M., PONGE, D., DEMIR, E., et al., "Orientation gradients and geometrically necessary dislocations in ultrafine grained dual-phase steels studied by 2D and 3D EBSD", Materials Science and Engineering A, v. 527, pp. 2738-2746, Apr. 2010.

[9] TAKAYAMA, Y., SZPUNAR, J.A., KATO, H., "Analysis of intergranular misorientation related to deformation in an Al-Mg-Mn alloy", Materials Science Forum, v. 495-497, pp. 1049-1054, Sep. 2005.

[10] BIELER, T.R., EISENLOHR, P., ROTERS, F., et al., "The role of heterogeneous deformation on damage nucleation at grain boundaries in single phase metals", International Journal of Plasticity, v. 25, pp. 1655-1683, Sep. 2009.

[11] ROLLETT, A.D., BRAHME, A.P., ROBERTS, C.G., "An overview of accomplishments and challenges in recrystallization and grain growth", Materials Science Forum, v. 558-559, pp. 33-42, Oct. 2007.

[12] POUILLIER, E., GOURGUES, A.-F., TANGUY, D., et al., "A study of intergranular fracture in an aluminum alloy due to hydrogen embrittlement", International Journal of Plasticity, v. 34, pp. 139-153, Jul. 2012.

[13] ABUZAID, W.Z., SANGID, M.D., CARROLL, J.D., et al., "Slip transfer and plastic strain accumulation across grain boundaries in Hastelloy X", Journal of the Mechanics and Physics of Solids, v. 60, n. 6, pp. 1201-1220, Jun. 2012.

[14] BIELER, T.R., EISENLOHR, P., ZHANG, C., et al., "Grain boundaries and interfaces in slip transfer", Current Opinion in Solid State and Materials Science, v. 18, n. 4, pp. 212-226, Aug. 2014.

[15] BADIOLA, J., IZA-MENDIA, A., GUTIÉRREZ, I., "Evaluation of intragranular misorientation parameters measured by EBSD in a hot worked austenitic stainless steel", Journal of Microscopy, v. 228, n. 3, pp. 373-383, Nov. 2007.

[16] WAGNER, F., ALLAIN-BONASSO, N., BERBENNI, S., et al., "On the Use of EBSD to Study the Heterogeneity of Plastic Deformation”, Materials Science Forum, v. 702-703, pp. 245-252, Jan. 2012.

[17] GITHINJI, D.N., NORTHOVER, S., BOUCHARD, P.J., et al., "An EBSD study of the deformation of service-aged 316 austenitic steel”, Metallurgical and Materials Transactions A, v. 44, n. 9, pp. 4150-4167, Sep. 2013.

[18] SCHAYES, C., BOUQUEREL, J., VOGT, J.B., et al., "A comparison of EBSD based strain indicators for the study of Fe-3Si steel subjected to cyclic loading", Materials Characterization, v. 115, pp. 61-70, May 2016. 
[19] SUBEDI, S., POKHAREL, R., ROLLETT, A.D., "Orientation gradients in relation to grain boundaries at varying strain level and spatial resolution”, Materials Science and Engineering A, v. 638, pp. 348-356, Jun. 2015.

[20] TAKAYAMA, Y., SZPUNAR, J.A., "Stored Energy and Taylor Factor Relation in an Al-Mg-Mn Alloy Sheet Worked by Continuous Cyclic Bending”, Materials Transactions, v. 45, n. 7, pp. 2316-2325, May 2004.

[21] WRIGHT, S.I., NOWELL, M.M., FIELD, D.P., “A Review of Strain Analysis Using Electron Backscatter Diffraction”, Microscopy and Microanalysis, v. 17, n. 3, pp. 316-329, Jun. 2011.

[22] MOUSSA, C., BERNACKI, M., BESNARD, R., et al., "About quantitative EBSD analysis of deformation and recovery substructures in pure Tantalum", In: 36th Riso International Symposium on Materials Science, IOP Conf. Series: Materials Science and Engineering, v. 89, pp. 012038, Sep. 2015.

[23] WRIGHT, S.I., FIELD, D.P., NOWELL, M,M,, "Post processing effects on GND calculations from EBSD-based orientation measurements", In: 36th Riso International Symposium on Materials Science, IOP Conf. Series: Materials Science and Engineering, v. 89, pp. 012049, Sep. 2015.

[24] KAMAYA, M., “Assessment of local deformation using EBSD: Quantification of accuracy of measurement and definition of local gradient”, Ultramicroscopy, v. 111, n. 8, pp. 1189-1199, Jul. 2011.

[25] KAMAYA, M., “Assessment of local deformation using EBSD: Quantification of local damage at grain boundaries", Materials Characterization, v. 66, pp. 56-67, Apr. 2012.

[26] VACHHANI, S.J., DOHERTY, R.D., KALIDINDI, S.R., "Studies of grain boundary regions in deformed polycrystalline aluminum using spherical nanoindentation", International Journal of Plasticity, v. 81, pp. 87-101, Jun. 2016.

[27] WAGNER, F., OUAREM, A., GU, C.F., ALLAIN-BONASSO, N., et al., "A new method to determine plastic deformation at the grain scale", Materials Characterization, v. 92, pp. 106-117, Mar. 2014. 